\title{
A Robust Zonal Fractal Dimension Method for the Recognition of Handwritten Telugu Digits
}

\author{
MSLB. Subrahmanyam \\ Research Scholar, JNTU Kakinada, Kakinada, 533001, India \\ Email: subrahmanyam_mtech@rediffmail.com \\ V. Vijaya Kumar \\ Director, Directorate of Admissions, Professor \& Head, Dept. of Computer Science, \\ Rayalaseema University, Kurnool, India \\ Email: drvvk144@gmail.com \\ B. Eswara Reddy \\ Professor of CSE Dept. \& Principal of JNTU-A College of Engineering, Kalikiri,India \\ Email: eswarcsejntu@gmail.com
}

Received: 17 April 2018; Accepted: 14 June 2018; Published: 08 September 2018

\begin{abstract}
Recognition of handwritten digits is most challenging sub task of character recognition due to various shapes, sizes, large variation in writing styles from person to person and also similarity in shapes of different digits. This paper presents a robust Telugu language handwritten digit recognition system. The Telugu language is most popular and one of classical languages of India. This language is spoken by more than 80 million people. The proposed method initially performs preprocessing on input digit pattern for removing noise, slat correction, size normalization and thinning. This paper divides the preprocessed Telugu handwritten digits into four differential zones of $2 \times 2,3 \times 3$, $4 \times 4$ and $6 \times 6$ pixels and extracts 65 features using Fractal dimension (FD) from each zone. The proposed zonal fractal dimension (ZFD) method uses, Feed forward backward propagation neural network (FFBPNN) for classifying the digits with learning rate of 0.01 and sigmoid function as an activation function on extracted 65 features. This paper evaluated the efficiency of the proposed method based on 5000 Telugu handwritten digit samples, each consists of ten digits from different groups of people and totally 50,000 samples. The performance of classification of the proposed method also evaluated using statistical parameters like recall, precision, Fmeasure and accuracy.
\end{abstract}

Index Terms-Indian languages, zones, Fractal dimension and Feed forward backward propagation neural network

\section{INTRODUCTION}

Optical character recognition (OCR) is one of important image processing techniques which converts an input image into corresponding editable text as an output. These systems provide an interface between human and machine for automation of text documents with lot of time saving and human effort. Initially OCR was started for recognizing digits and characters in machine printed images after that extended to recognize in handwritten images. Handwritten recognition is always challenging task due to various shapes, sizes, writing variability between individuals and even variability of writing style of individuals with the environment as well as state of mind, mood of the person, writing instruments etc. Offline and online are two different categories in Handwritten recognition systems. In offline systems image text is processed once document scanned completely while in online systems processing is done during stroke made by the writer on digital surface using optical pen. One of the subtasks of handwritten character recognition problem is handwritten digit recognition, which is aimed to identify a numeric value within the document. It has specific applications in automatic pin code or zip code reading of postal system, bank cheques processing, vehicle number recognition, interpretation of ID numbers and so on.

The feature extraction and classification are two very important steps of handwritten numeric recognition to achieve high accuracy in this process. Spatial and transform domain are two major categories in Feature extraction techniques. Invariant moments [1], projections $[2,64]$, zoning feature [3], an adaptive zoning techniques $[4],[5],[55]$ and [6],gradient based features [7][8][10] are some of examples of spatial domain techniques. In spatial domain statistical or structural or both [9-13] properties can be used for extracting features. In transform domain Fourier transform (FT) [14-16], Gabor transform [17-22], Wavelet transform [23-26] are some for extracting features in the literature. Probabilistic neural network [27], template matching [65-67], Artificial neural network [28, 29, 56, 68], Hidden Markov-model [30, 31], support vector machine (SVM) [32], [11] are some classification techniques for recognizing numerals or scripts. 
Abdelhak Boukharouba et al. [32] proposed a method using transition information in vertical and horizontal directions with Freeman chain code features and SVM classifier to recognize Persian handwritten numerals. In [33] Ritesh Sarkhl et al. proposed an architecture for recognizing top most Indian scripts. In [34] Ritesh Sarkhel1 et al presented multi-objective approach to recognize isolated Bangla characters and numerals. Kalyan Sourav Dash[35] et al proposed a novel method for Odia numerals recognition in transformed domain using non-redundant stockwell transform as well as Slantlet transform features. In [36] D. Impedovo et al. presented a paper on survey of zoning methods for handwritten character recognition. In [37] Ehsan Mohebi et al. proposed method for classification of handwritten digits using convolutional recursive modified selforganizing map. In [38] Kalyan S Dash et al proposed method for recognizing Odia numerals using Kirsch gradient operator features. In this method they used Modified Quadratic Discriminant Function (MQDF), Discriminative Learning Quadratic Discriminant Function (DLQDF) classifiers for achieving high accuracy. Thomas Deselaers [39] et al. presented handwritten digit classification using latent log-linear models. Nibaran Das [40] Proposed methodology for extracting features using Genetic algorithm from seven sets of local regions with SVM classifier. In [41] Nibaran Das et al. introduced a new combination of features using principle component analysis for numeral recognition of five Indian sub-continent scripts using SVM classifier. In [42] Xiao-Xiao Niu n, ChingY.Suen presented a hybrid method for digits recognition using trainable features from convolutional neural network and SVM recognizer. In [43] Ghulam Ali Montazer proposed numeral recognition engine for Farsi script using neural network and fuzzy theory.

This paper proposes a robust method for recognizing handwritten Telugu digits, which uses zone-based feature extraction technique using FD and FFBPNN for classification.

This paper is organized as follows Section II gives details about Telugu script characteristics, section III gives related work, section IV contains the description of our proposed method,Section V contains experimental results, Section VI contains discussion of the results from the proposed method and conclusions are given in Section VII.

\section{TElugu SCRIPT CHARACTERISTISCS}

India is country that have vast number of languages and officially recognized eighteen languages for regular use. Telugu is one of classical languages within these and is widely spoken Dravidian language. Telugu script used for writing the Telugu language. This script also used for writing some of minority languages such as Chenchu, Savara and Manna-Dora in South India. This script consists of 16 vowels denote as "achchus", 36 consonants denote as "hallus" and totally consists of 52 letters. And also several semi-vowel symbols called "maatras' and half consonants called 'voththus'. Combining all these together roughly provide 100 basic orthographic units. Fig.1. shows image of Telugu alphabets. Telugu script also contains ten digits as shown in Fig. 2.

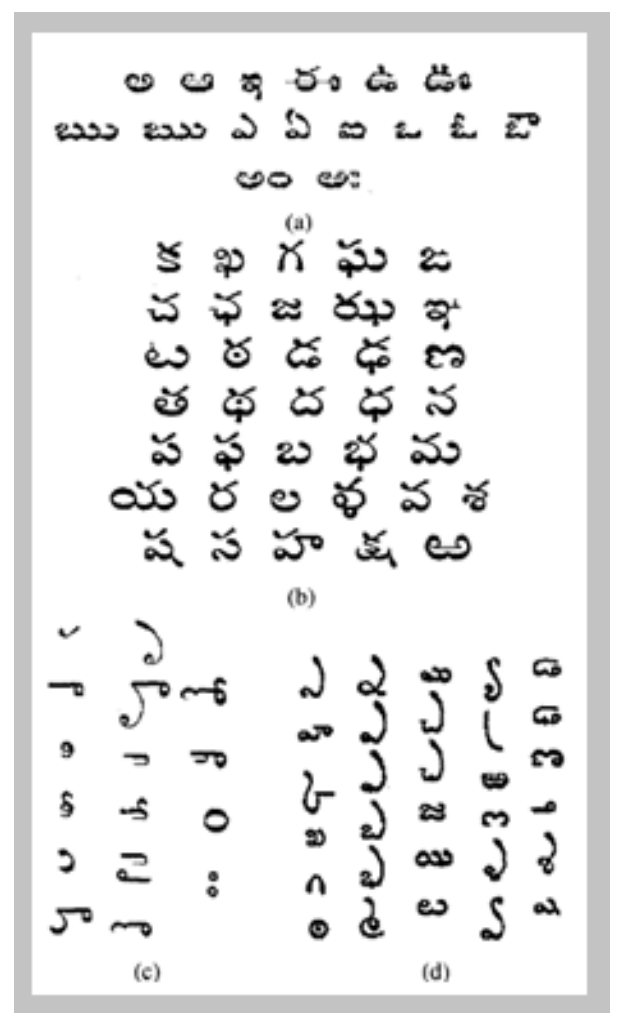

Fig.1. Telugu Alphabet ( a) Achchlu (b) Halulu (c ) Maatras ( d) Voththulu.

\section{$0093 \&$ \& $2 v \varepsilon$}

Fig.2. Telugu Numerals

\section{RELATED WORK}

OCR for Indian languages in general is more complex than those of European languages due to the linguistic complexity of large number of vowels, consonants and various different combinations of vowels and consonants. Relatively less work has been reported for the Indian scripts. In [44] Akhilesh Pandey et al. proposed off-line handwritten Hindi numeral recognition using majority voting scheme for extracting features and classified using neural network. V. Rajashekarraradhya et al proposed using zone based features for recognizing four south Indian scripts in [45]. In [46] Benne R.G have proposed for Kannada Devanagari and Telugu scripts handwritten digits recognition using structural features and recognized using k-nearest neighbor algorithm. In [47] B.V.Dhandra et at proposed method for recognizing three scripts using global and local structural features and classified using probabilistic neural network. In [48] S.M.Mali proposed handwritten Marathi numeral recognition using density and central moments. S.M.Mali [49] proposed for recognizing isolated Marathi numeral 
using SVM classifier with, Fourier Descriptor and chain codes as features. In [50] G. Rajput et al. proposed method using features from Chain codes and Fourier descriptor with SVM classifier to recognize Kannada printed and handwritten mixed numerals. Survey on Kannada digits recognition proposed by $\mathrm{C}$. Hallur in [51].For recognizing Handwritten Gurmukhi Character Recognition Gita Sinha [52] proposed a method using zone based feature extraction and classified with SVM. In [53] Shivanand Killedar et al proposed using convolution technique for recognizing Handwritten Kannada numerals. Sunnetha [54] have proposed a method for classification of off-line handwritten numerals of Telugu, Tamil and Kannada Indian scripts. In [55] Pratibha Singh et al proposed a reliable approach for Devanagari numeral recognition. Using Convolutional NN in [56] M. A. H. Akhand et al proposed a method for recognizing numerals of isolated Bangali as well as English Mixed documents. In [55] M Radhika et al proposed using features from Haar wavelet transform with boundary Moment invariants for Telugu Numerals recognition. Ch. N. Manisha [56] provided the information and comparative analysis on various methods for recognizing Telugu characters and numerals. In [57] J Jyothi et al. proposed a method using features from Discrete Wavelet Transform (DWT) and SVM classifier for Telugu numerals recognition. In [58] C.V.Chakradhar et al. presented a study and various methods to recognize the Online Handwritten Character Recognition in Telugu Language. In [59] U Ravi Babu proposed a method using features from structural, skeleton and water reservoir for recognizing Telugu digits.

\section{METHODOLOGY}

Telugu Handwritten digit recognition is more challenging task because of similar looking shapes, sizes and lot of variation between different writing styles. These challenging tasks effects not only recognition accuracy but also leads to recognition complexity. Telugu handwritten digits samples of ten users are shown in Fig.3. Fig.4. shows the block diagram of ZFD model and various steps of the ZFD model is given below.

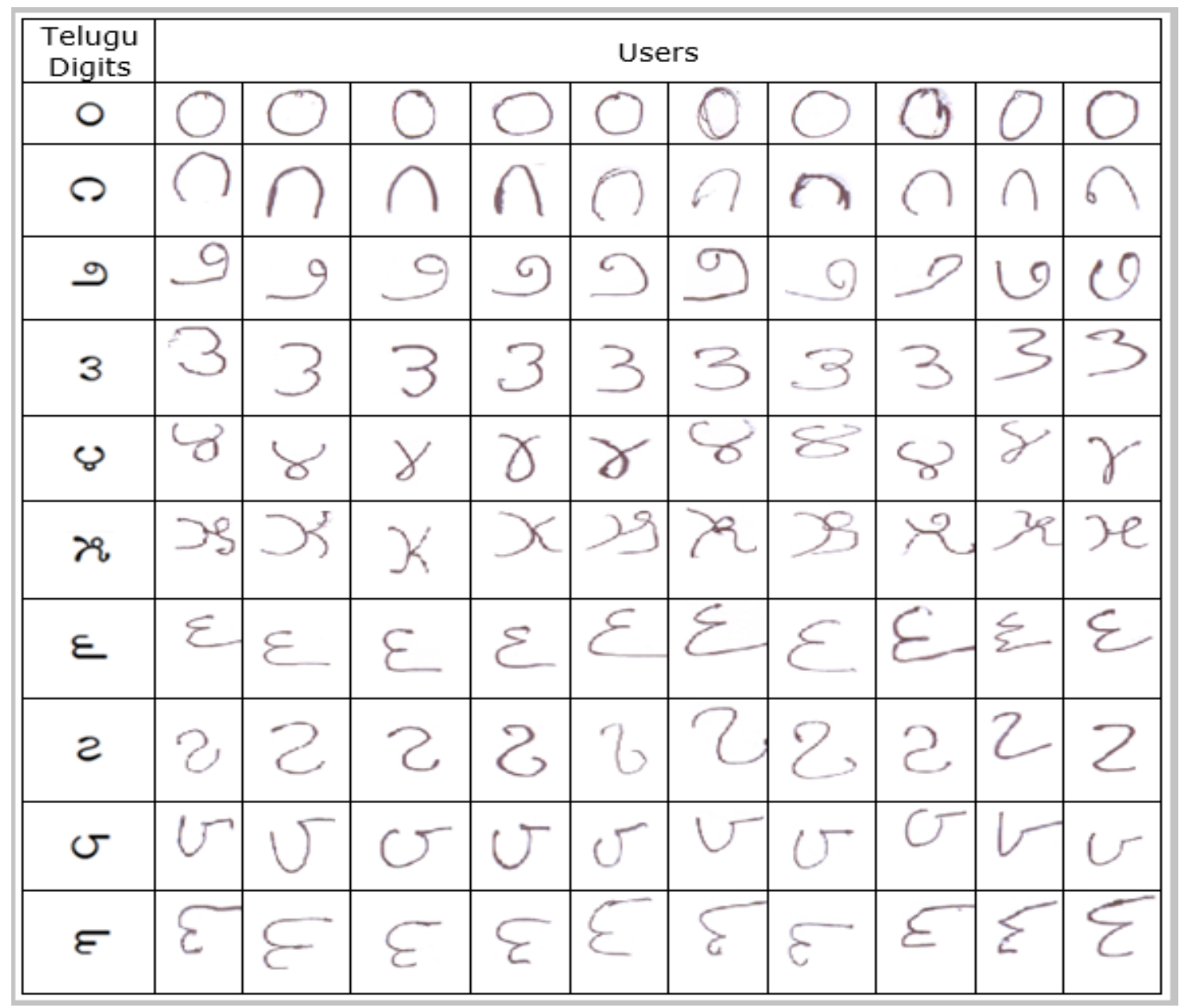

Fig.3. Handwritten Telugu digits of Ten users. 


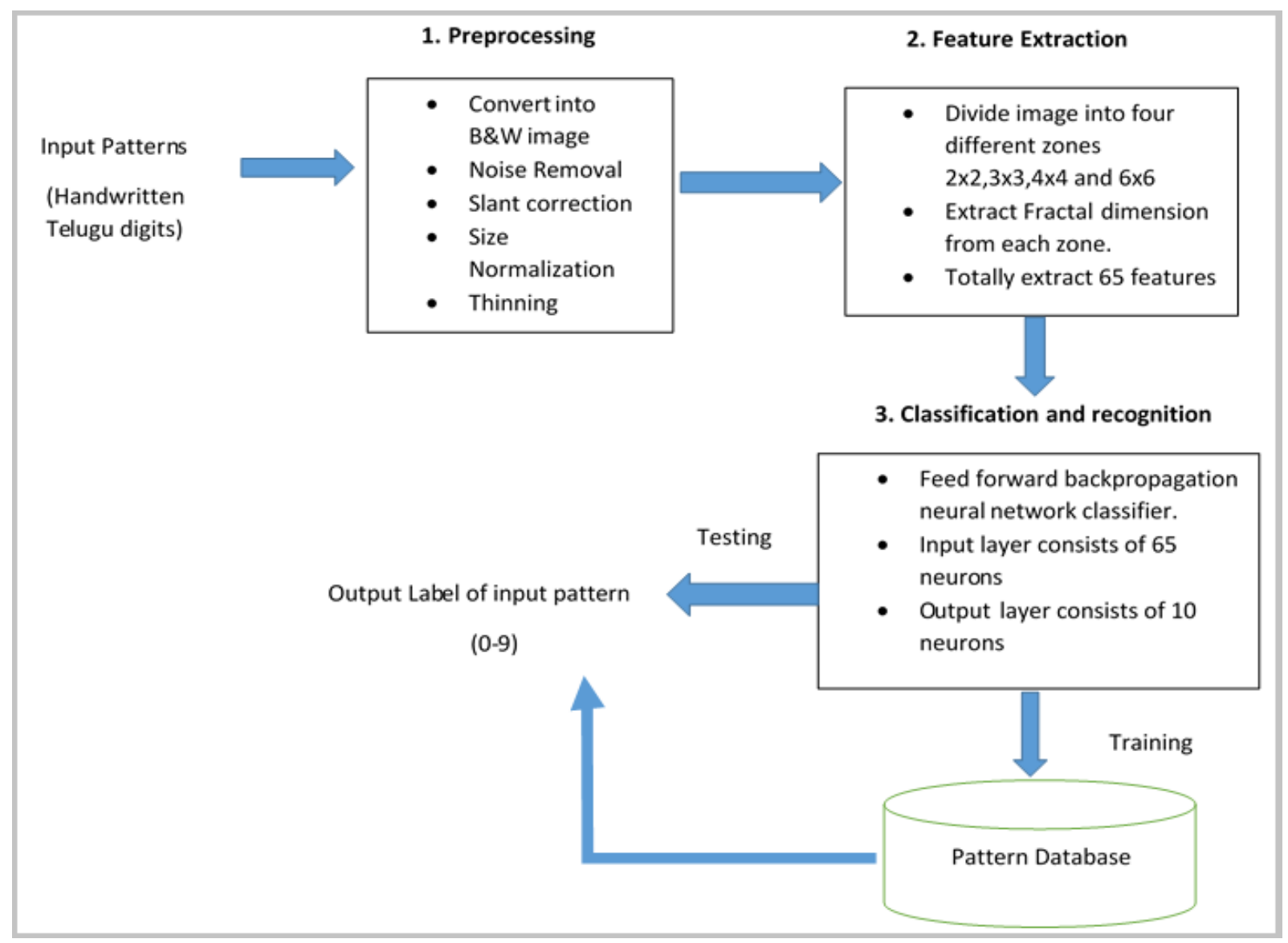

Fig.4. Block diagram of proposed robust ZFD method.

\section{Preprocessing:}

To enhance significant features and to suppress unwanted distortions of the input handwritten Telugu digit images the present paper used a pre-processing technique in step 1 .

In the preprocessing process, this paper initially converted the given color or grayscale handwritten digit image into black and white image using Otsu's [62] threshold. Fig.5 (b) shows the output of the input image 5(a) after converting into black and white image. To remove noise from input image 5(a), median filter is employed and the output is shown in Fig 5(c). One of the crucial variations in handwritten digits is due to slant. After in-depth study from different users and their writing styles we observed that slant angle alpha $(\alpha)$ would be in the range $\left[-45^{\circ} 45^{\circ}\right]$. To remove this the proposed ZFD model derived the following algorithm.

Algorithm for removing slant:

Begin

1. Initialize the $\alpha$ with $45^{\circ}$ and let $\mathrm{A}$ be the original image

2. While $\alpha>1$ do steps 3 and 4

3. Now apply sheer transform on given image with angel's $\alpha / 2$ and $-\alpha / 2$. Let $B$ and $C$ be the corresponding images respectively.

4. Find the minimum width image from $\mathrm{A}, \mathrm{B}$ and $\mathrm{C}$. and make it as A.

5. Let $\alpha$ be $\alpha / 2$ and go to step 2 .
End.

One of the major problems in handwritten scripts is the size of the each alphabet and it depends on user to user and the same user may write with different sizes. To deal this aspect the proposed research derived a normalization process. To normalize the input numeral image, the proposed ZFD derived the following algorithm and by this, digits are normalized to a size of $48 \times 48$ pixels. Fig. 6 (a) shows normalized image from Fig.5 (d).

\section{Algorithm for normalization of size:}

Let $\mathrm{W}$ denote width and $\mathrm{H}$ denote height of the original image. Let $\mathrm{NR}_{\mathrm{W}}$ and $\mathrm{NR}_{\mathrm{H}}$ be the width and height after normalization and equal to 48 and 48 respectively i.e. $\mathrm{NR}_{\mathrm{W}}=48$ and $\mathrm{NR}_{\mathrm{H}}=48$.

Begin:

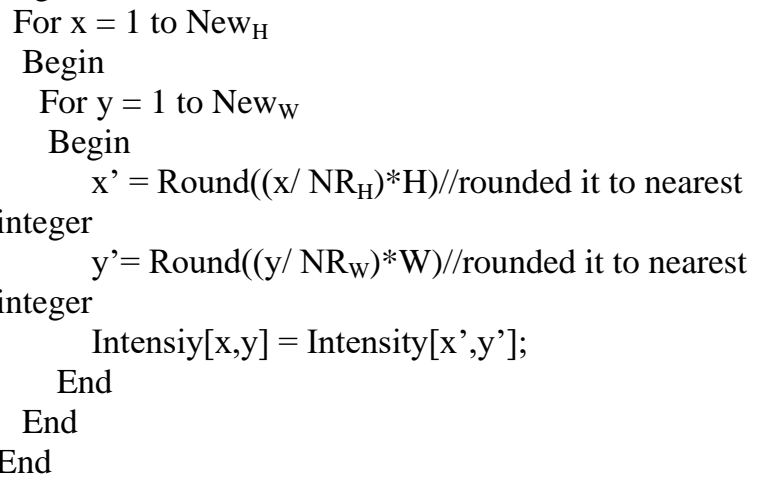


To extract shape information of the digit the proposed ZFD method uses T.Y.Zang [63] thinning algorithm on given handwritten digit image. Fig.6 (b) represents image after applying thinning algorithm on Fig.6 (a).

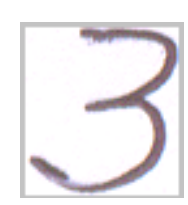

(a)

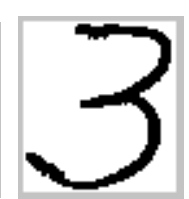

(b)

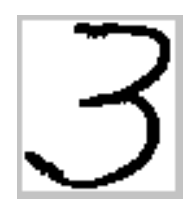

(c)

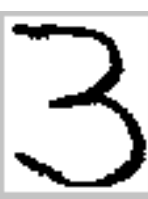

(d)
Fig.5. (a) Input handwritten Telugu 'three' digit image (b) Image after applying Otsu's algorithm on (a). (c) Image after noise removal from b (d) Image after slant correction from c.

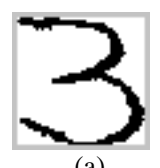

(a)

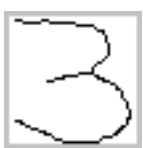

(b)
Fig.6. (a) Image after normalization of 5(d). (b) Image after applying thinning algorithm on (a).

\section{Feature Extraction:}

Feature extraction is very important step for extracting potential features to achieve high recognition accuracy. Efficient features are always have similar values for objects belong to same category and distinct for different categories. Obtaining features from handwritten images always difficult task due to high degree of variation between persons. Also since different person write the same numeric with different styles and strokes which are not smooth and irregular in nature. To address the above and to derive significant, precise and robust features from the handwritten numeric data the proposed ZFD utilizes fractal theory. Fractal theory overcome the limitations of Euclidean geometry with mathematical value and can be applied to characterize irregular or complex figures. We can quantify the irregularity and complexity of objects by using fractal analysis with a measurable value called fractal dimension (FD), which provides new approach to shape characterization. FD contains information about their geometric structure with non-integer dimension. The proposed ZFD uses box covering method for calculating the FD of the object. This method initially subdivide the object into a number of small boxes using the given scaling factors and computes FD from each box as shown in the Fig.7.

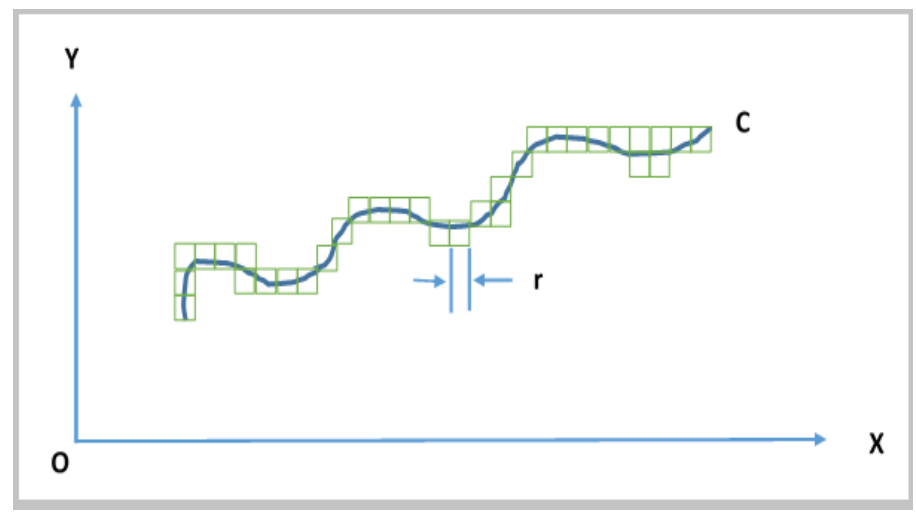

Fig.7. Box counting method for curve C.

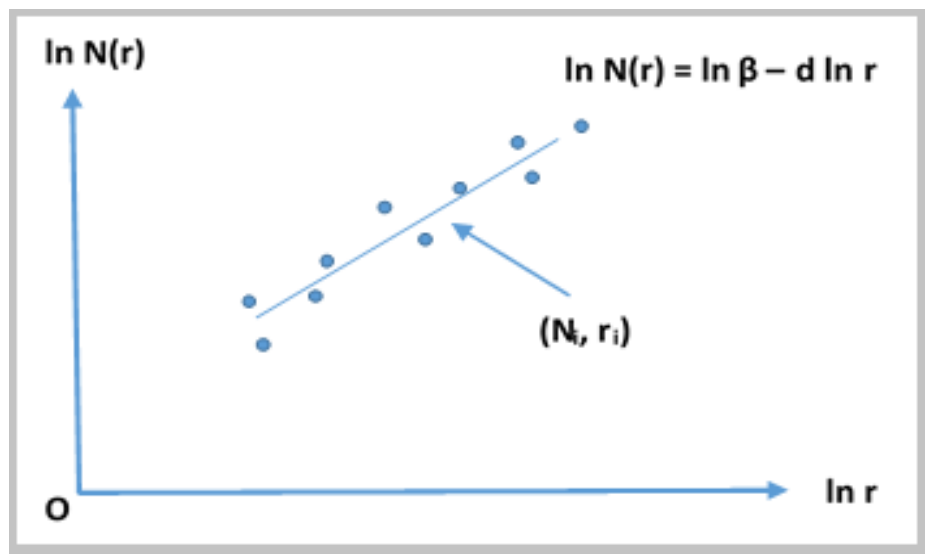

Fig.8. Graphical representation for the calculation of FD.

The FD ' $d$ ' of a geometric object is defined by using equation (1) and can estimate the FD d of a geometric object by performing a logarithmic linear regression using equation (2).

$$
\mathrm{d}=\lim _{r \rightarrow 0} \frac{[\ln N(r)]}{\left[\ln \left(\frac{1}{r}\right)\right]}
$$


Where $N(r)$ is the number of boxes covering the object and $r$ is the size of the box.

$$
\ln \mathrm{N}(\mathrm{r})=\ln \beta-\mathrm{d} * \ln \mathrm{r}
$$

where $\{\ln \beta\}$ is $y$-intersect.

Figure 8 shows the graphical representation for the calculation of FD by taking $\{\ln r\}$ as $x$-axis and $\{\ln N(r)\}$ as $y$-axis. From this figure we can estimate FD from the slope of best fitting line.

To extract features the proposed method uses zonebased approach by dividing the normalized image into $2 \times 2,3 \times 3,4 \times 4$ and $6 \times 6$ pixel zones horizontally and vertically respectively as shown in the Fig.9. From each zone the proposed method extracts fractal dimension using box counting method.

The proposed method extracted 4,9,16 and 36 FD features from $2 \times 2,3 \times 3,4 \times 4$ and $6 \times 6$ normalized zonal Telugu handwritten digit images respectively. This leads to a total of 65 features. Fig.9. shows different zones of normalized Telugu handwritten digit "Three"

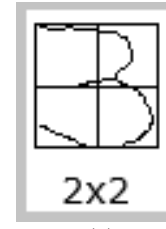

(a)

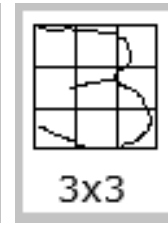

(b)

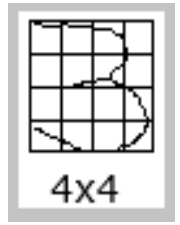

(c)

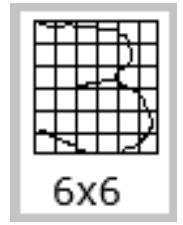

(d)
Fig.9. Normalized Telugu digit zonal images (a) $2 \times 2$ zone (b) $3 \times 3$ zone (c) $4 \times 4$ zone (d) $6 \times 6$ zone.

\section{Classification and recognition using artificial neural} network.
The classification stage is very important to assign label from features extracted in the previous stage. The extracted features are given as the input to the classification process. To classify the handwritten digit image the proposed method uses the FFBPNN. There are two distinct stages using FFBP network to process data, a training stage and a classification stage. The architecture of proposed model consists of one input layer and one output layer as shown in Fig.10. The input layer consists of 65 neurons corresponds to each feature vector and output layer consists of 10 neurons each corresponds to 10 Telugu handwritten numerals that have to be identified. The proposed method uses delta rule for updating weights with learning rate equal to 0.01 and sigmoid function as an activation function as given in equation (3).

Let $\mathrm{w}_{\mathrm{ki}}$ denote $\mathrm{k}^{\text {th }}$ neuron $\mathrm{i}^{\text {th }}$ weight and the delta rule for $\mathrm{k}$ using activation function $\mathrm{s}(\mathrm{x})$ given by

$$
\Delta \mathrm{w}_{\mathrm{ki}}=\alpha\left(\mathrm{t}_{\mathrm{k}}-\mathrm{y}_{\mathrm{k}}\right) \mathrm{s}^{\prime}\left(\mathrm{h}_{\mathrm{k}}\right) \mathrm{x}_{\mathrm{i}}
$$

Where

$\alpha$ denote learning rate which is equal to 0.01 .

$\mathrm{s}(\mathrm{x})$ denote neuron's activation function and $\mathrm{s}(\mathrm{x})=\frac{1}{1+e^{-x}}$ $\mathrm{s}^{\prime}(\mathrm{x})$ represents derivative of $\mathrm{s}(\mathrm{x})$ and mathematically equal to $(1-\mathrm{s}(\mathrm{x})) \mathrm{s}(\mathrm{x})$

$\mathrm{t}_{\mathrm{k}}$ denote target output

$\mathrm{h}_{\mathrm{k}}$ denote weighted sum of the neuron's outputs

$\mathrm{y}_{\mathrm{k}}$ denote actual output

$\mathrm{X}_{\mathrm{i}}$ is the $\mathrm{i}^{\text {th }}$ input

$\mathrm{y}_{\mathrm{k}}=\mathrm{s}\left(\mathrm{h}_{\mathrm{k}}\right)$ and $\mathrm{h}_{\mathrm{k}}=\operatorname{sigma}\left(\mathrm{x}_{\mathrm{i}} \mathrm{W}_{\mathrm{ki}}\right)$.

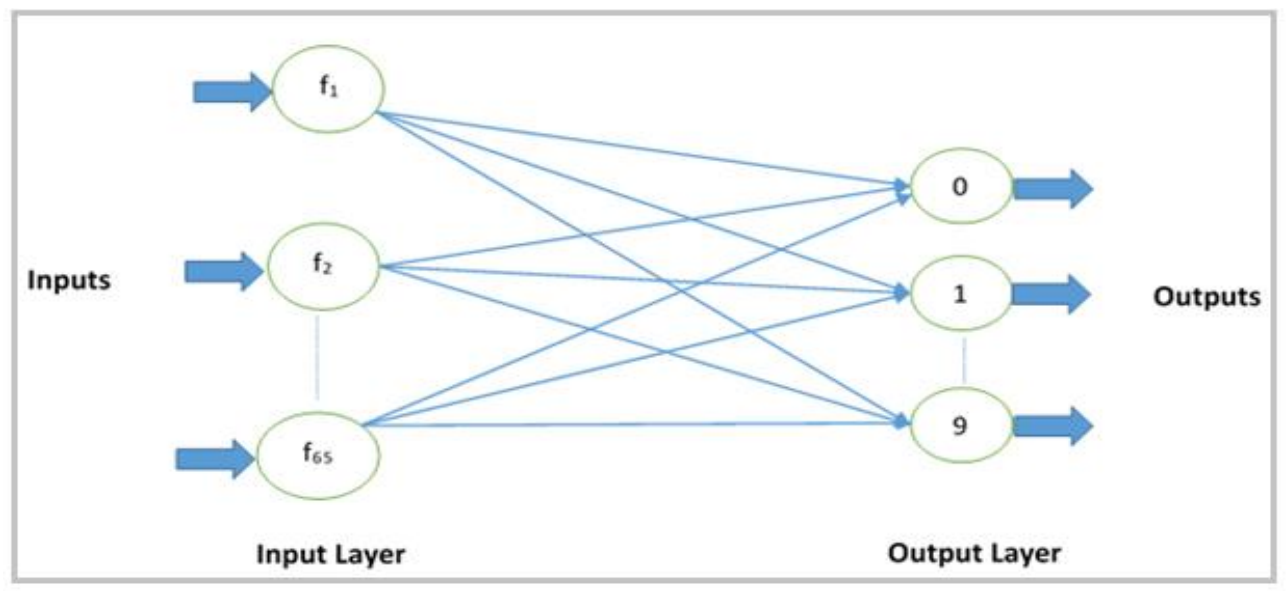

Fig.10. Architecture of proposed NN.

\section{RESUlTS}

In this section we experimented the benefits of propped ZFD model for the Telugu handwritten digits recognition. This paper considered a total of 50,000 samples of Telugu language handwritten digits which consists of ten digits (0-9) from each 5000 individuals. The samples are collected from individuals like students, house wives, employees etc. Out of these 50,000 samples 30,000 samples are considered for training and remaining 20,000 are considered for testing. To extract features initially the proposed ZFD model experimented the accuracy by dividing the normalized image into different zones like $2 \times 2,3 \times 3,4 \times 4$ and $6 \times 6$ and extracted FD from each one. The proposed ZFD model achieved $89 \%$ of accuracy when considering only $2 \times 2$ with four features. The proposed ZFD model achieved $94 \%$ accuracy when 
considering only $6 \times 6$ with 36 features. The proposed ZFD model also experimented with the combination of different zones. The proposed ZFD model achieved $96 \%$ accuracy from the combination of $2 \times 2,3 \times 3$ and $4 \times 4$ with 29 features. The proposed ZFD achieved excellent accuracy of 99.23 with the combination of $2 \times 2,3 \times 3,4 \times 4$ and $6 \times 6$ with 65 features. The proposed ZFD extracted 65 features from each numeral image using fractal dimension. FFBPNN has been used for training above dataset with 65 neurons as input layer and 10 neurons as output numerals with learning rate 0.01 . The recognition accuracy of each digit is listed out in Table 1 and shows $99.23 \%$ of average accuracy. Fig.11. shows the comparison of accuracy of all digits along with average accuracy using proposed method.

Table 1. Recognition Accuracy of each Numeral

\begin{tabular}{|c|c|c|c|c|c|c|c|c|c|c|c|}
\hline Digit & Zero & One & Two & Three & Four & Five & Six & Seven & Eight & Nine & Avg \\
\hline percentage & 100 & 99.15 & 99.24 & 98.55 & 99.3 & 98.75 & 98.65 & 99.35 & 99.5 & 98.85 & 99.23 \\
\hline
\end{tabular}

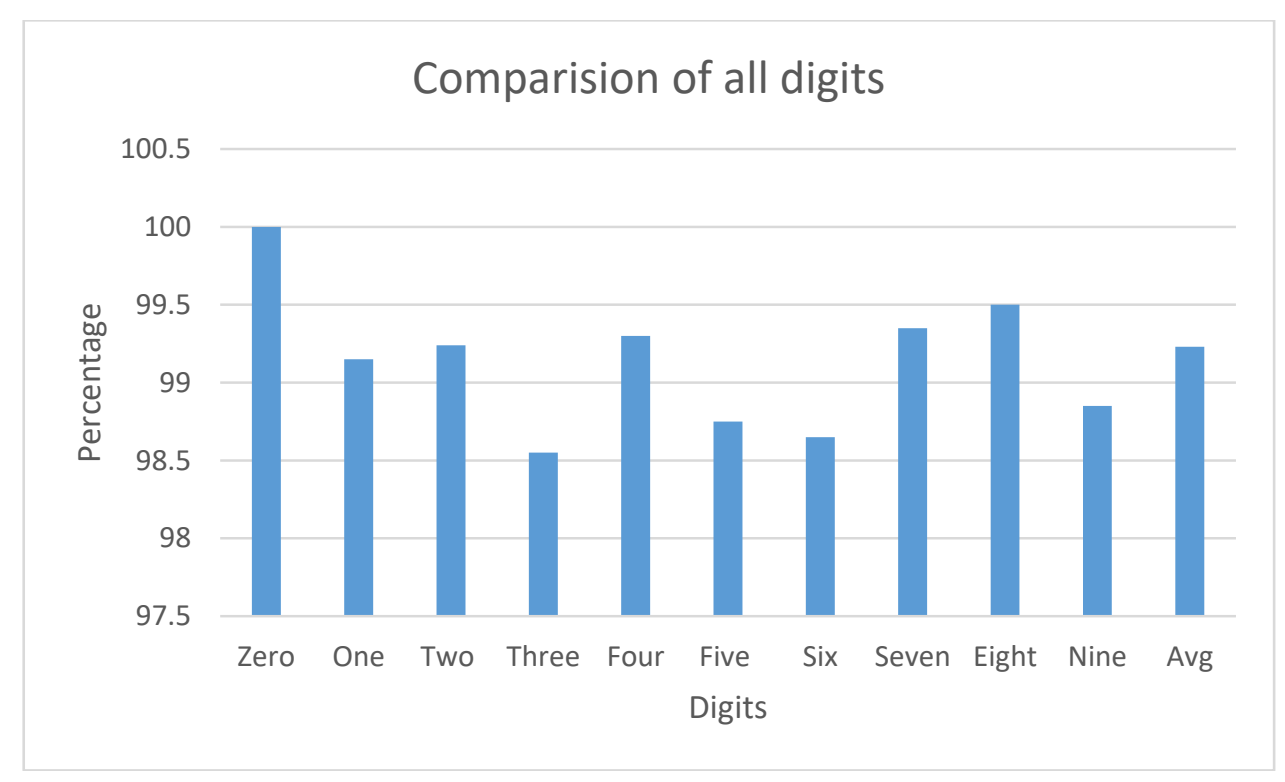

Fig.11. Comparison of average accuracy of all digits.

Table 2 shows the misclassification between all handwritten Telugu digits from the proposed ZFD method. No misclassification happened for digit 0 and totally 173 misclassifications happened for remaining digits from 1 to 9 . Out of 173 misclassifications more than $60.1 \%$ i.e. 104 misclassifications happened from the group of digits 3, 5, 6 and 9. In this group more misclassification happened for digit 3 and less misclassification happened for digit 9.Remaining $39.9 \%$ i.e. 69 misclassifications happened from remaining digits $1,2,4,7$ and 8 . In this group of digits maximum misclassification happened for 1 and less misclassification happened for digit 8 .

Table 2. Misclassification between digits.

\begin{tabular}{|c|c|c|}
\hline Digit & $\begin{array}{c}\text { No of times } \\
\text { misclassified }\end{array}$ & Percentage \\
\hline 0 & 0 & 0 \\
\hline 1 & 17 & 9.82 \\
\hline 2 & 15 & 8.67 \\
\hline 3 & 29 & 16.76 \\
\hline 4 & 14 & 8.09 \\
\hline 5 & 25 & 14.45 \\
\hline 6 & 27 & 15.60 \\
\hline 7 & 13 & 7.51 \\
\hline 8 & 10 & 5,78 \\
\hline 9 & 23 & 13.29 \\
\hline All & 173 & 100 \\
\hline
\end{tabular}

Table 3 shows the results of misrecognition of the handwritten Telugu digits from the proposed ZFD method. From the table we can observe that $65.87 \%$ i.e. 114 misrecognitions happened from group of digits $0,1,2,8$ and 9 . In this group more misrecognition happened for digit 0 i.e. twenty nine times and eighteen times misrecognition happened for digit 9. And also 59 misrecognition out of 173 with $34.13 \%$ happened for remaining group of digits $3,4,5,6$ and 7 . In this group more misrecognition happened for digit 7 and less happened for 5. Fig.12 shows the comparison between misclassification and misrecognition of handwritten

Table 3. Misrecognition between digits.

\begin{tabular}{|c|c|c|}
\hline Digit & $\begin{array}{c}\text { No of times } \\
\text { misrecognized }\end{array}$ & Percentage \\
\hline 0 & 29 & 16.76 \\
\hline 1 & 26 & 15.02 \\
\hline 2 & 21 & 12.13 \\
\hline 3 & 11 & 6.35 \\
\hline 4 & 9 & 5.20 \\
\hline 5 & 8 & 4.62 \\
\hline 6 & 14 & 8.09 \\
\hline 7 & 17 & 9.82 \\
\hline 8 & 20 & 11.56 \\
\hline 9 & 18 & 10.40 \\
\hline All & 173 & 100 \\
\hline
\end{tabular}


Telugu digits from 0 to 9 using proposed ZFD model. Fig.13 represents some of samples which are confused digits from the proposed ZFD method. This image also contains the truth label and predicted label from the proposed ZFD method.

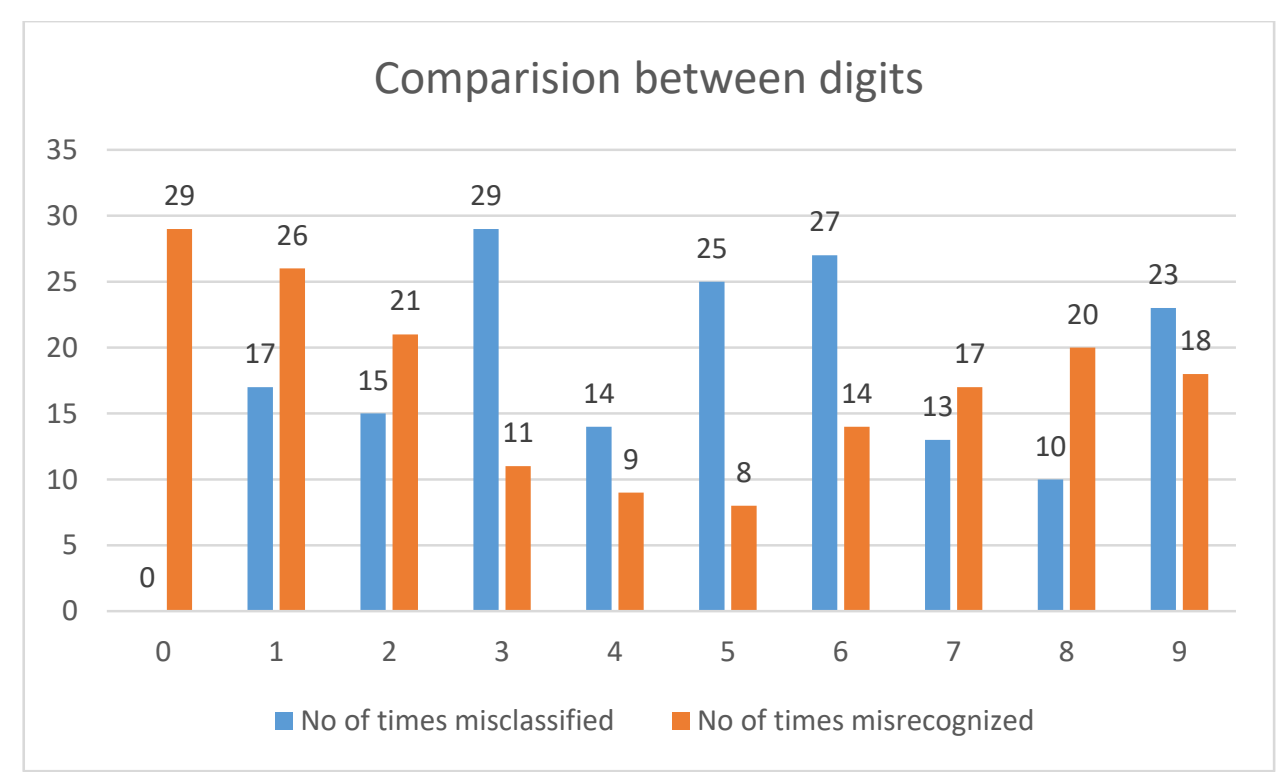

Fig.12. Comparison of misclassification and misrecognition between digits from proposed ZFD model.

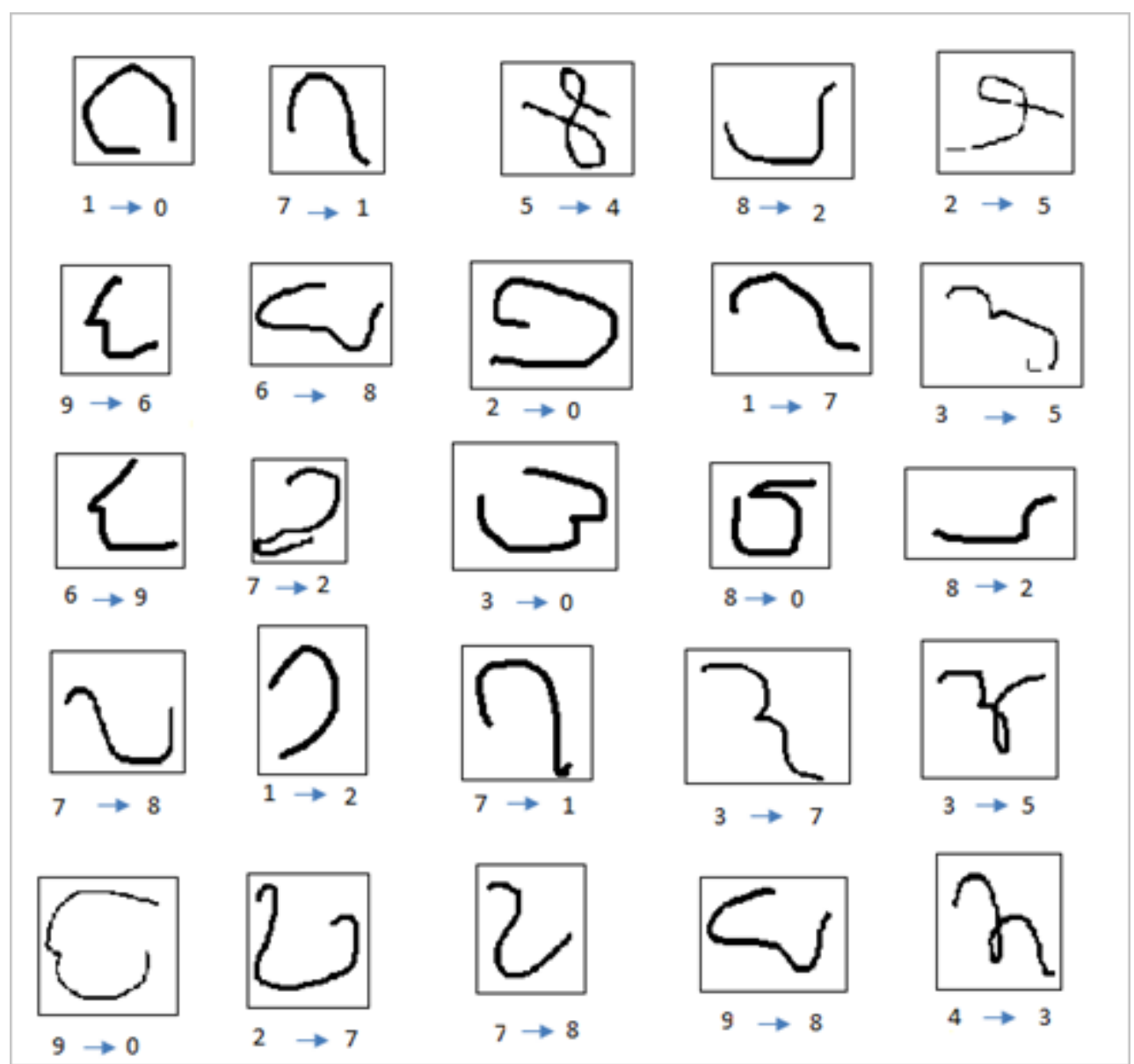

Fig.13. Sample images confused by proposed ZFD method. Each box represents handwritten Telugu digit and lower labels of image represents corresponding truth -> perdition. 


\section{A. Performance measures for classification}

We evaluated proposed ZFD model performance using confusion matrix and Table 4 shows confusion matrix obtained from proposed ZFD model. In this paper, Precision (P), Recall (R), Accuracy (A) and F-measure (F) are used to evaluate the performance efficiency as given in the equation 4.The performance measures for classification of proposed ZFD method for Telugu handwritten digits is listed out in Table 5 and Fig.14 shows the comparison of performance measures of all digits.

Mathematically we evaluate these statistical methods using following procedure.

$$
\begin{gathered}
\text { Precision }(\mathrm{P})=\frac{T P}{T p+F P} \\
\operatorname{Recall}(\mathrm{R})=\frac{T P}{T p+F N} \\
\text { Accuracy }(\mathrm{A})=\frac{T P+T N}{T P+T N+F P+F N} \\
\mathrm{~F}-\operatorname{Measure}(\mathrm{F} 1)=\frac{2 * P * R}{P+R}
\end{gathered}
$$

Where

- $\quad \mathrm{TF}$ denote number of correct predictions that an instance is negative.

- FN denote number of incorrect of predictions that an instance negative.

- FP denote number of incorrect predictions that an instance is positive.

- TP denote number of correct predictions that an instance is positive.

From Table 4 and Table 5 we can observe that digit 0 achieved maximum value in case of recall, F-measure and accuracy with values equal to $1,0.9927$ and 0.9987 respectively. But in case of precision digit 5 achieved maximum value which is equal to 0.9959 . Digit 4 and Digit 0 achieved minimum values in case of recall and precession respectively. Digit 1 achieved minimum value in case of F-measure and accuracy with values equal to 0.9892 and 0.9979 respectively.

Table 4. Confusion Matrix for all digits

\begin{tabular}{|c|c|c|c|c|c|c|c|c|c|c|c|}
\hline Digits/Digits & 0 & 1 & 2 & 3 & 4 & 5 & 6 & 7 & 8 & 9 & Recall \\
\hline 0 & 2000 & 0 & 0 & 0 & 0 & 0 & 0 & 0 & 0 & 0 & 1 \\
\hline 1 & 7 & 1983 & 4 & 1 & 0 & 0 & 0 & 2 & 3 & 0 & 0.9915 \\
\hline 2 & 2 & 3 & 1985 & 3 & 0 & 3 & 1 & 1 & 2 & 0 & 0.9924 \\
\hline 3 & 2 & 2 & 9 & 1971 & 1 & 3 & 0 & 8 & 3 & 1 & 0.9855 \\
\hline 4 & 3 & 3 & 2 & 1 & 1986 & 0 & 2 & 2 & 1 & 0 & 0.993 \\
\hline 5 & 4 & 12 & 0 & 1 & 1 & 1975 & 0 & 3 & 0 & 4 & 0.9875 \\
\hline 6 & 1 & 1 & 5 & 3 & 1 & 0 & 1973 & 1 & 3 & 12 & 0.9865 \\
\hline 7 & 4 & 3 & 1 & 0 & 1 & 0 & 0 & 1987 & 3 & 1 & 0.9935 \\
\hline 8 & 4 & 1 & 0 & 0 & 5 & 0 & 0 & 0 & 1990 & 0 & 0.995 \\
\hline 9 & 2 & 1 & 0 & 2 & 0 & 2 & 11 & 0 & 5 & 1977 & 0.9985 \\
\hline Precision & 0.9857 & 0.9870 & 0.9895 & 0.9944 & 0.9954 & 0.9959 & 0.9929 & 0.9915 & 0.9900 & 0.9909 & \\
\hline
\end{tabular}

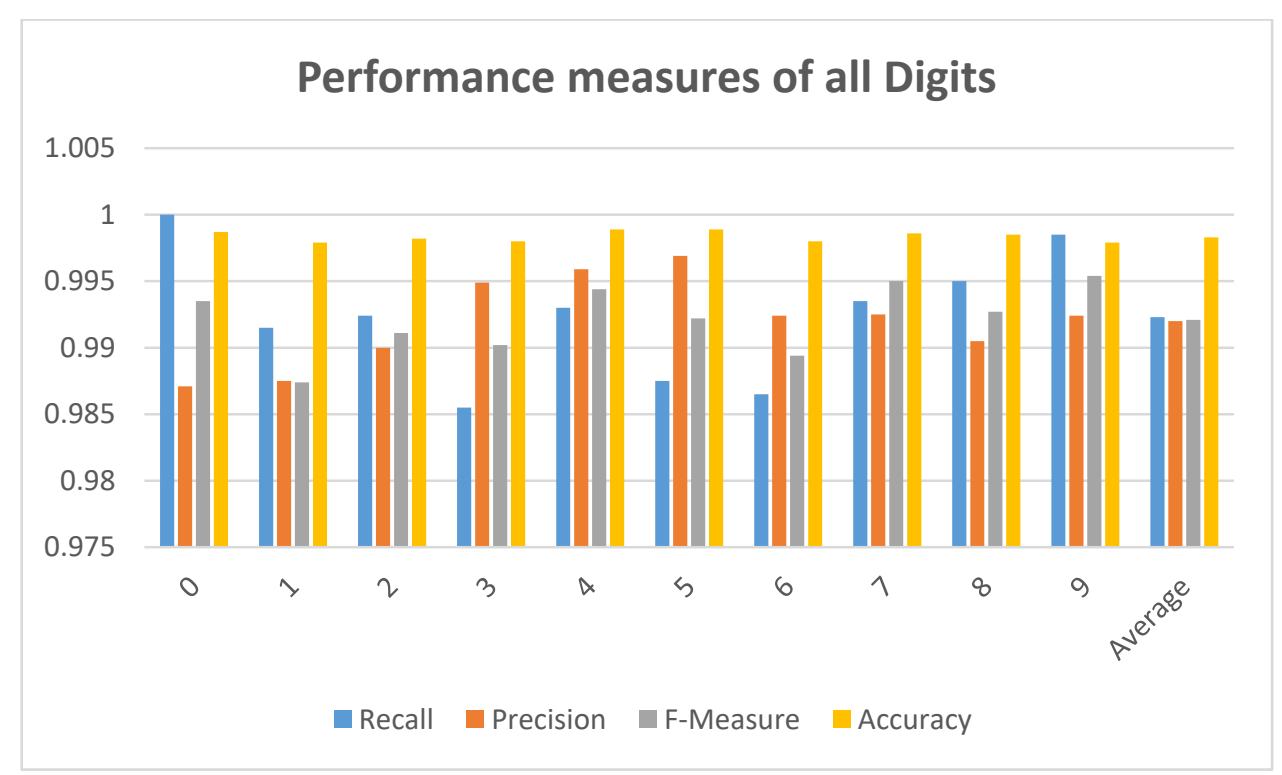

Fig.14. Comparison of performance measures of all digits. 
Table 5. Performance measures for classification

\begin{tabular}{|c|c|c|c|c|}
\hline Class/Digits & Recall & Precision & F-Measure $\left(\mathrm{F}_{1}\right)$ \\
\hline 0 & 1 & 0.9857 & 0.9927 & 0.9892 \\
\hline 1 & 0.9915 & 0.9870 & 0.9909 & 0.9979 \\
\hline 2 & 0.9924 & 0.9895 & 0.9899 & 0.9982 \\
\hline 3 & 0.9855 & 0.9944 & 0.998 & 0.9916 \\
\hline 4 & 0.993 & 0.9954 & 0.9896 & 0.9924 \\
\hline 5 & 0.9875 & 0.9959 & 0.9989 & 0.998 \\
\hline 6 & 0.9865 & 0.9929 & 0.9915 & 0.9989 \\
\hline
\end{tabular}

\section{B. Comparative analysis with different methods:}

This paper compared proposed ZFD model with other existing methods for Telugu handwritten digit recognition using S. V. Rajashekararadhya et al[45], B.V.Dhandra et al [47], S. M. Mali [48], G. Hemantha
Kumar et al.[51],G. G. Rajput et at [52] Gita Sinha et at. [54] Algorithms and these methods achieved 98.6\%, 98\%, $97,89 \%, 98 \%, 97.76 \%$ and $95.11 \%$ respectively. Authors in [45] and [54] are considered zonal features like our proposed method ZFD. But proposed SVM for

Table 6. Comparison with the other methods

\begin{tabular}{|l|l|l|l|}
\hline Method & Features & Classification & Accuracy in \% \\
\hline $\begin{array}{l}\text { S.V. } \\
\text { RAJASHEKARARADHYA } \\
\text { et al[45] }\end{array}$ & zone-based feature extraction & SVM & 98.6 \\
\hline B.V.Dhandra et al.[47] & $\begin{array}{l}\text { global and local structural } \\
\text { features }\end{array}$ & Probabilistic Neural Network & 98 \\
\hline S. M. Mali[48] & density and central moments & SVM & 97.89 \\
\hline G. Hemantha Kumar et al.[51] & $\begin{array}{l}\text { local intensity order patter } \\
\text { descriptor }\end{array}$ & $\begin{array}{l}\text { five-fold cross validation } \\
\text { technique with numeral network } \\
\text { classifier }\end{array}$ & 98 \\
\hline G. G. Rajput et at.[52] & Fourier descriptors & SVM & 97.76 \\
\hline Gita Sinha et at.[54] & $\begin{array}{l}\text { Zone Based Hybrid Feature } \\
\text { Extraction }\end{array}$ & SVM & 95.11 \\
\hline Proposed & Fractal Dimension & NN & 99.23 \\
\hline
\end{tabular}

\section{Accuracy in \%}

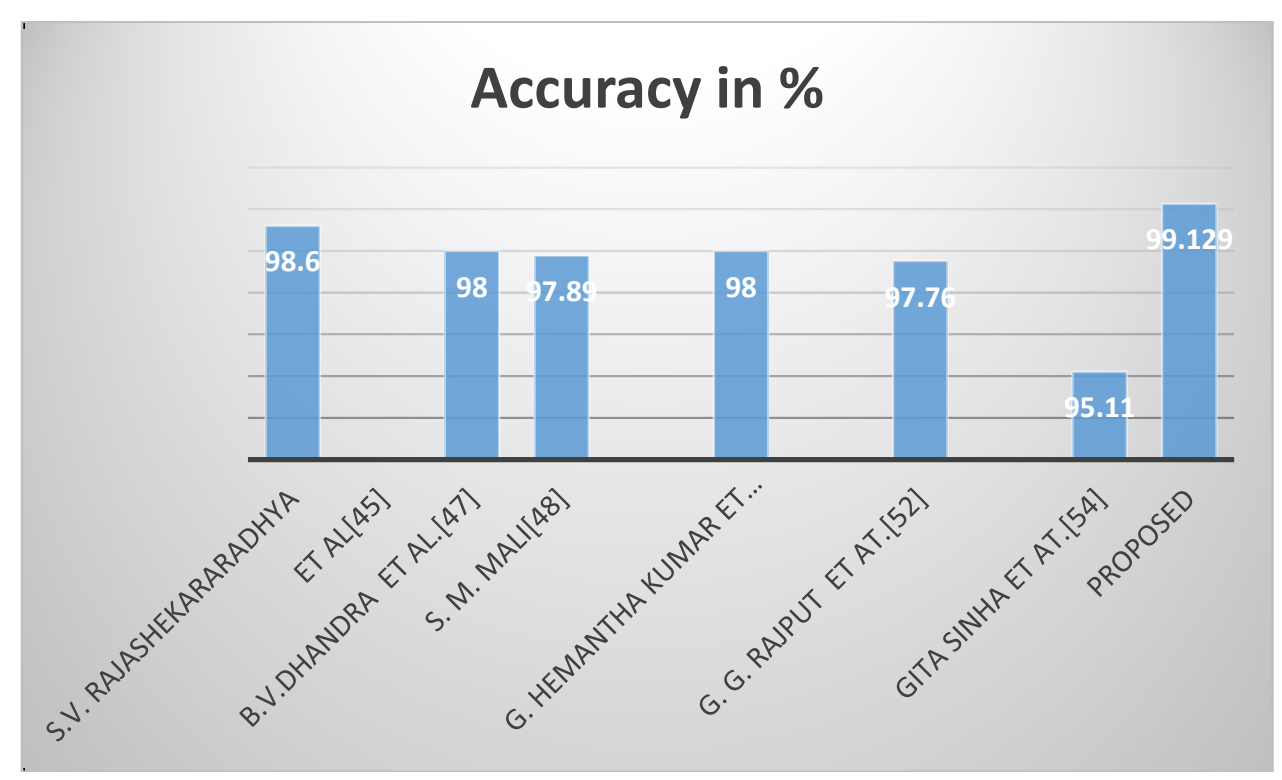

Fig.15. Comparison of proposed method with other methods. 
classification. In [47] and [51] authors proposed classification using neural network like our proposed method ZFD. Our method achieved 99.23\% accuracy. The detailed comparison results are shown in the following Table 6 and Fig.15. From the table we can observe that the proposed method achieved very good results compared to all other methods. These results reveal that the proposed ZFD method is very suitable for Telugu Numerals recognitions.

\section{DISCUSSION}

Experimental results reveal $100 \%$ accuracy for digit zero and almost good results in case of digits 1, 2, 4, 7, 8 and 9 (more than 99\%) from current proposed ZFD model. Out of total 20000 in training set, there are only 173 samples that are not successfully recognized. We have got an accuracy of $100 \%$ on the training samples $(30,000$ samples) and also an accuracy of $100 \%$ when the training is made on the whole dataset (50,000 samples). No misclassification happened for digit 0 with all other digits but 29 misrecognitions happed with remaining digits from 1 to 9 . Out of 29 misrecognitions $7,2,2,3,4,1,4,4$ and 2 misrecognitions happened with digits $1,2,3,4,5,6,7,8$ and 9 respectively. From this we can observe that maximum confusion happened for digit 0 with digit 1 seven times. For digit 1 maximum misrecognitions happened with digit 5 and maximum misclassification happened with digit 0 . Also no misclassification happened with digits 4, 5, 6 and 9 .

In case of digit 2 , totally eleven misrecognitions and fifteen misclassifications happened with remaining digits respectively. And also no misrecognition happened with digits $0,5,8$ and 9 and no misclassification happened with digits 4 and 9. Maximum nine misrecognitions happened with digit 3.For digit 3 no misrecognition happened with 0,7 and 8 . Maximum nine times misclassification happened with digit 2 but no misclassification happened with digit 6 . In case of digit 4 no misrecognition happened with $0,1,2$ and 9 and also no misclassification with digits 5 and 9 . For digit 5 neither misrecognition nor misclassification with digit 6 and maximum confusion happened with digit 1.For digit 6 no misrecognition happened with $0,1,3,7$ and 8 and no misclassification happened with digit 6 . And also maximum 11 times misrecognition happened with digit 9 . In case of digit 7 no misrecognition happened with 0,8 and 9 and no misclassification happened with 3, 5 and 6. Maximum eight times misrecognition happened with 3 . For digit 8 no misrecognition happened with 0 and 5 and no misclassification happened 2,3,5,6, and 9. But maximum five times misrecognition happened with digit 9. In case of digit 9 no misrecognition happened with digits $0,1,2,4$ and 8 and no misclassification with digits 4 and 7. Maximum twelve times misrecognition happened with digit 6 .

From confusion table we observed that more confusion happened between 6 and 9.No confusion happened between digits 5 and 6, 4 and 9, 8 and 5 and between 2 and 9.
The proposed ZFD method minimized the overall confusion between the digits and improved accuracy because proposed method extracts features in zonal wise using FD. This indicates that the proposed method very suitable for recognizing Telugu numerals.

\section{CONCLUSION}

This Paper introduced a robust method for recognizing handwritten Telugu digits. After an in-depth study on the Telugu handwritten digits we observed that the zonal based features are most important and crucial factor for achieving high accuracy. Based on this observation this paper proposed a robust ZFD method for recognition of handwritten Telugu numerals by dividing image into four different kind of zones $2 \times 2,3 \times 3,4 \times 4$ and $6 \times 6$. Totally 65 features were extracted from each zone using FD and FFBPNN used for classification. The experimental analysis revealed the effectiveness of the proposed ZFD model. This paper evaluated the classification performance using statistical methods Precision, recall, accuracy and $F_{1}$. The proposed method can also be extended to other Indian languages like Kannada, Malayalam, Tamil, and Hindi etc.

\section{REFERENCES}

[1] C.H. The, R.T. Chin, "On image analysis by the methods of moments", IEEE Trans. Pattern Anal. Mach. Intell. 10 (1988) 496-513.

[2] A.L. Koerich, "Unconstrained handwritten character recognition using different classification strategies", in: Proceedings of International Workshop on Artificial Neural Networks in Pattern Recognition, 2003.

[3] L.S. Oliveira, R. Sabourin, F. Bortolozzi, C.Y. Suen, "Automatic recognition of handwritten numerical strings: a recognition and verification strategy", IEEE Trans. Pattern Recogn. Mach. Intell.24 (11) (2002) 1438-1454.

[4] S. Impedovo, F.M. Mangini, G. Pirlo, "A new adaptive zoning technique for handwritten digit recognition", in: ICIAP, 2013.

[5] F.M. Mangini, G. Pirlo, "Adaptive zoning design by supervised learning using multi-objective optimization", Int. J. Comput.Intell. Appl. 13 (01) (2014).

[6] D. Impedovo and G. Pirlo, "Zoning methods for handwritten character recognition: A survey",„ Pattern Recognit., vol. 47, no. 3, pp. 969-981, 2014.

[7] C.L. Liu, "Normalization-cooperated gradient feature extraction for handwritten character recognition", IEEE Trans. Pattern Anal. Mach. Intell. 29 (8) (2007).

[8] C.L. Liu, C.Y. Suen, "A new benchmark on the recognition of handwritten Bangla and Farsi numeral characters", Pattern Recogn. 42 (2009) 3287-3295.

[9] Trier, O.D., Jain, A.K., Taxt, T.: "Feature extraction methods for character recognition - a survey", Pattern Recognition., 1996, 29, (4), pp. 641-662.

[10] Shi, M., Fujisawa, Y., Wakabayashi, T., Kimura, F.: "Handwritten numeral recognition using gradient and curvature of gray scale image", Pattern Recognit., 2002, 35, (10), pp. 2051-2059.

[11] Jain, A.K., Duin, R.P.W., Mao, J.,"Statistical pattern recognition: a review", IEEE Trans. Pattern Anal. Mach. Intell., 2000, 22, (1), pp. 4-37. 
[12] Yang, M., Kidiyo, K., Ronsin, J.: "A survey of shape feature extraction techniques",in Yin, P. (Ed.): 'Pattern recognition techniques, technology and applications', 2008, pp. 43-90.

[13] Liu, C., Nakashima, K., Sako, H., Fujisawa, H.: "Handwritten digit recognition:benchmarking of state-ofthe-art techniques", Pattern Recognit., 2003, 36, (10),pp. 2271-2285.

[14] Shridhar, M., Badreldin, A., "High accuracy character recognition algorithm using Fourier and topological descriptor", Pattern Recognit., 1984, 17, (5), pp. 515-524.

[15] Mahmoud, S.A., "Arabic character recognition using Fourier descriptors and character contour encoding", Pattern Recognit., 1994, 27, (6), pp. 815-824.

[16] Bourbakis, N.G., Gumahad, A.T., "Knowledge based text character recognition using Fourier transform". Proc. Second Int. IEEE Conf. on Artificial Intelligence, 1990, pp. 571-576.

[17] Mutelo, R.M., Woo, W.L., Dlay, S.S., "Discriminant analysis of the two-dimensional Gabor features for face recognition", IET Comput. Vis., 2008, 2,(2), pp. 37-49.

[18] Whichello, A.P., Yan, H., "Reconstruction of character skeletons using Gabor filter features", Electron. Lett., 1995, 31, (22), pp. 1911-1912.

[19] Hamamoto, Y., Uchimura, S., Watanabe, M., Yasuda, T., Tomita, S., "A Gabor filter-based method for recognizing handwritten numerals", Pattern Recognit.,1998, 31, (4), pp. 395-400.

[20] Huo, Q., Ge, Y., Feng, Z.-D., "High performance Chinese OCR based on Gabor features, discriminative feature extraction and model training". Proc. ICASSP, Utah, 2001, vol. 3, pp. 1517-1520.

[21] Wang, X., Ding, X., Liu, C.”Optimized Gabor filter based feature extraction for character recognition". Proc. 16th ICPR, Quebec, Canada, 2002, vol. 4, pp. 223-226.

[22] Liu, C., Koga, M., Fujisawa, H., "Gabor feature extraction for character recognition: comparison with gradient feature". Proc. Eighth Int. Conf. on Document Analysis and Recognition, 2005, vol. 1, pp. 121-125.

[23] Bhattacharya, U., Chaudhuri, B.B., "Handwritten numeral databases of Indian scripts and multistage recognition of mixed numerals", IEEE Trans. Pattern Anal. Mach. Intell., 2009, 31, (3), pp. 444-457.

[24] Mowlaei, A., Faez, K., Haghighat, A.T., "Feature extraction with wavelet transform for recognition of isolated handwritten Farsi/Arabic characters and numerals". Proc. $14^{\text {th }}$ Int. Conf. on Digital Signal Processing, 2002, vol. 2, pp. 923-926.

[25] Huang, L., Huang, X.,"Multiresolution recognition of offline handwritten Chinese characters with wavelet transform". Proc. Sixth Int. Conf. Document Analysis and Recognition, Seattle, 2001, pp. 631-634.

[26] Kunte, R.S., Samuel, R.D.S., "Script independent handwritten numeral Recognition". Proc. IET Int. Conf. VIE, Bangalore, 2006, pp. 94-98.

[27] F.A. Al-Omari, O. Al-Jarrah, "Handwritten Indian numerals recognition system using probabilistic neural networks", Adv Eng. Inform. 18 (2004) 9-16.

[28] Y. LeCun and Y. Bengio, "Word-level training of a handwritten word recognizer based on convolutional neural networks," in International Conference on Pattern Recognition, 1994, p. 88-92.

[29] S. Basu, N. Das, R. Sarkar, M. Kundu, M. Nasipuri, and D. K. Basu, "An MLP based Approach for Recognition of Handwritten 'Banglae Numerals," pp. 407-417, Mar. 2012.
[30] A.de s.britto Jr, R. Sabourin, F. Bortolozzi, and C. Y. Suen, "The recognition of handwritten numeral strings using a two-stage HMM-based method," Int. J. Doc. Anal. Recognit., vol. 5, no. 2-3, pp. 102-117, 2003.

[31] A. Vinciarelli, S. Bengio, and H. Bunke, "Offline recognition of unconstrained handwritten texts using HMMs and statistical language models.," IEEE Trans. Pattern Anal. Mach. Intell., vol. 26, no. 6, pp. 709-20, Jun. 2004

[32] Abdelhak Boukharouba, Abdelhak Bennia "Novel feature extraction technique for the recognition of handwritten digits" Saudi Computer Society, King Saud University, Applied Computing and Informatics (2017) 13, 19-26 .

[33] Ritesh Sarkhel, Nibaran Das, Aritra Das, Mahantapas Kundu, Mita Nasipuri, “A Multi-scale Deep Quad Tree Based Feature Extraction Method for the Recognition of Isolated Handwritten Characters of popular Indic Scripts", Pattern Recognition (2017), doi: 10.1016/j.patcog.2017.05.022.

[34] Ritesh Sarkhel, Nibaran Das, Amit K. Saha and Mita Nasipuri, "A multi-objective approach towards cost effective isolated handwritten Bangla character and digit recognition", Pattern Recognition, http://dx.doi.org/10.1016/j.patcog.2016.04.010.

[35] Kalyan Sourav Dash, Niladri B. Puhan, Ganapati Panda, "Handwritten numeral recognition using non-redundant Stockwell transform and bio-inspired optimal zoning" IET Image Process., 2015, Vol. 9, Iss. 10, pp. 874-882, doi: 10.1049/iet-ipr.2015.0146 www.ietdl.org

[36] D. Impedovo, D. Impedovo "Zoning methods for handwritten character recognition: A survey" Pattern Recognition

(2013), http://dx.doi.org/10.1016/j.patcog.2013.05.021i.

[37] Ehsan Mohebi, Adil Bagirov, "A convolutional recursive modified Self Organizing Map for handwritten digits recognition" Neural Networks 60 (2014) 104-118, http://dx.doi.org/10.1016/j.neunet.2014.08.001.

[38] Kalyan S Dash, N. B. Puhan and Ganapati Panda, "A Hybrid Feature and Discriminant Classifier for High Accuracy Handwritten Odia Numeral Recognition" 9781-4799-2027-3/14/\$31.00 @2014 IEEE.

[39] Thomas Deselaers, Tobias Gass, Georg Heigold, and Hermann Ney "Latent Log-Linear Models for Handwritten Digit Classification" Ieee Transactions On Pattern Analysis And Machine Intelligence, VOL. 34, NO 6, JUNE 2012

[40] Nibaran Das, Ram Sarkar, Subhadip Basu, Mahantapas Kundu, Mita Nasipuri*, Dipak Kumar Basu, "A genetic algorithm based region sampling for selection of local features in handwritten digit recognition application" Applied Soft Computing 12 (2012) 1592-1606, doi:10.1016/j.asoc.2011.11.030.

[41] Nibaran Das, Jagan Mohan Reddy, Ram Sarkar, Subhadip Basu, Mahantapas Kundu, Mita Nasipuri*,Dipak Kumar Basu "A statistical-topological feature combination for recognition of handwritten numerals" Applied Soft Computing $12 \quad$ (2012) 2486-2495, doi:10.1016/j.asoc.2012.03.039.

[42] Xiao-Xiao Niu n, ChingY.Suen, "A novel hybrid CNNSVM classifier for recognizing handwritten digits" Pattern Recognition 45 (2012) 1318-1325, doi:10.1016/j.patcog.2011.09.021.

[43] Gholam Ali Montazer a,*, Hamed Qahri Saremi b, Vahid Khatibi a," A neuro-fuzzy inference engine for Farsi numeral characters recognition" Expert Systems with 
Applications $\quad 37 \quad$ (2010) 6327-6337, doi:10.1016/j.eswa.2010.02.088.

[44] Akhilesh Pandey Amresh Kumar 3. Rajiv Kumar ,"Handwritten Devanagri Number Recognition using Majority Voting Scheme", International Journal of Computer Science and Information Technology \& Security (IJCSITS), ISSN: 2249-9555 Vol. 2, No.3, June 2012.

[45] V. Rajashekararadhya,Dr P.Vanaja Ranjan,"Handwritten Numeral/Mixed Numerals recognition of South-Indian scripts: The zone based feature extraction method", Journal of Theoretical and Applied Information Technology.

[46] Benne R.G.1, Dhandra B.V.1 and Mallikarjun Hangarge ,"Tri-scripts handwritten numeral recognition: a novel approach", Advances in Computational Research, ISSN: 0975-3273, Volume 1, Issue 2, 2009, pp-47-51.

[47] B.V.Dhandra, R.G.Benne, Mallikarjun Hangarge ,"Kannada, Telugu and Devanagari Handwritten Numeral Recognition with Probabilistic Neural Network:A Novel Approach”, IJCA Special Issue on "Recent Trends in Image Processing and Pattern Recognition"2010.

[48] S. M. Mali ,"Moment and density based handwritten Marathi numeral recognition", Indian Journal of Computer Science and Engineering (IJCSE) Vol. 3 No.5 Oct-Nov 2012.

[49] S. M. Mali ,"Multiple Feature Extraction Methods for Handwritten Marathi Numeral Recognition", International Journal of Engineering Research \& Technology (IJERT) Vol. 2 Issue 10, October - 2013.

[50] G. G. Rajput, Rajeswari Horakeri, Sidramappa Chandrakant ,"Printed and Handwritten Mixed Kannada Numerals Recognition Using SVM", International Journal on Computer Science and Engineering Vol. 02, No. 05, 2010, 1622-1626.

[51] Vishweshwarayya C. Hallur, Avinash A. Malawade, Seema G. Itagi ,'Survey on Kannada Digits Recognition Using OCR Technique", International Journal of Advanced Research in Computer Engineering \& Technology (IJARCET) Volume 1, Issue 10, December 2012.

[52] Gita Sinha, Anita Rani, Prof. Renu Dhir, Mrs. Rajneesh Rani ,"Zone-Based Feature Extraction Techniques and SVM for Handwritten Gurmukhi Character Recognition”, International Journal of Advanced Research in Computer Science and Software Engineering Volume 2, Issue 6, June 2012.

[53] Shivanand Killedar, Satish Deshapande ,"Kannada Handwritten Numerals Recognition and Translation Using Template Matching", International Journal on Recent Technologies in Mechanical and Electrical Engineering (IJRMEE) ISSN: 2349-7947 Volume: 2 Issue: 62015.

[54] Suneetha , G. Hemantha Kumar ,Narendra $\mathrm{R}$,"Handwritten Numeral Recognition using Local Intensity Order Pattern of Popular South Indian Scripts", International Journal of Latest Research in Engineering and Technology (IJLRET) ISSN: 2454-5031 Volume 02 Issue 05 || May 2016 || PP. 41 - 44.

[55] Pratibha Singh, Ajay Verma and Narendra S. Chaudhari," Reliable Devanagri Handwritten Numeral Recognition using Multiple Classifier and Flexible Zoning Approach", I.J. Image, Graphics and Signal Processing, 2014, 9, 6168, Published Online August 2014 in MECS (http://www.mecs-press.org/), DOI: 10.5815/ijigsp.2014.09.08.
[56] M. A. H. Akhand, Mahtab Ahmed and M. M. Hafizur Rahman, “ Convolutional Neural Network based Handwritten Bengali and Bengali-English Mixed Numeral Recognition", I.J. Image, Graphics and Signal Processing, 2016, 9, 40-50, Published Online September 2016 in MECS (http://www.mecs-press.org/), DOI: 10.5815/ijigsp.2016.09.06.

[57] M. Radhika Mani, R. Kavitha Lakshmi ,'An Approach for Telugu Numeral Recognition by Moment Invariants in Wavelet Transform Domain", International Journal of Innovative Research in Computer and Communication Engineering Vol. 1, Issue 8, October 2013.

[58] Ch. N. Manisha, E. Sreenivasa Reddy, Y.K. Sundara Krishna ,"A Study on Recognition Methods of Telugu Numerals and Characters", International Journal of Emerging Technology in Computer Science \& Electronics (IJETCSE) ISSN: 0976-1353 Volume 11 Issue 5 NOVEMBER 2014.

[59] J . Jyothi, K. Manjusha, M. Anand Kumar and K. P. Soman ,'Innovative Feature Sets for Machine Learning based Telugu Character Recognition”, Indian Journal of Science and Technology, Vol 8(24), DOI: 10.17485/ijst/2015/v8i24/79996, September 2015.

[60] C.V.Chakradhar, 2 B.Rajesh, 3 M.Raghavendra Reddy ,"A Study on online Handwritten Telugu Character Recognition", International Journal of Science, Engineering and Technology Research (IJSETR) Volume 5, Issue 7, July 2016.

[61] U Ravi Babu1 Y V V Satyanarayana2 S. Marthu Perumal ,"Printed Telugu Numeral Recognition based on Structural, Skeleton and Water Reservoir Features", International journal of computers \& technology, Vol 10, No 72013.

[62] Nobuyuki Otsu (1979). "A threshold selection method from gray-level histograms". IEEE Trans. Sys., Man., Cyber. 9 (1): 62-66. doi:10.1109/TSMC.1979.4310076.

[63] T.Y. Zang and C.Y. Suen, "A fast parallel algorithm for thinning digital patterns", Comm. ACM 27 (3) (1984) 236-239.

[64] Srinivasa Rao A.V,"Segmentation of Ancient Telugu Text Documents", I.J. Image, Graphics and Signal Processing, 2012, 6, 8-14, Published Online July 2012 in MECS (http://www.mecs-press.org/),DOI: 10.5815/ijigsp.2012.06.02.

[65] N. Shobha Rani and Vasudev T and Pradeep C.H," A Performance Efficient Technique for Recognition of Telugu Script Using Template Matching", I.J. Image, Graphics and Signal Processing, 2016, 8, 15-23.

[66] Afaz Uddin Ahmed , Taufiq Mahmud Masum , Mohammad Mahbubur Rahman," Design of an Automated Secure Garage System Using License Plate Recognition Technique", I.J. Intelligent Systems and Applications, 2014, 02, 22-28.

[67] Sarbjit Kaur," An Automatic Number Plate Recognition System under Image Processing", I.J. Intelligent Systems and Applications, 2016, 3, 14-25, Published Online March 2016 in MECS (http://www.mecs-press.org/), DOI: 10.5815/ijisa.2016.03.02.

[68] Pratibha Singh, Ajay Verma and Narendra S. haudhari," Devanagri Handwritten Numeral Recognition using Feature Selection Approach, I.J. Intelligent Systems and Applications, 2014, 12, 40-47. Published Online November 2014 in MECS (http://www.mecs-press.org/), DOI: $10.5815 /$ ijisa.2014.12.06. 


\section{Authors' Profiles}

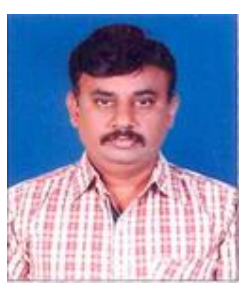

MSLB Subrahmanyam is working as a Head-imaging and algorithm practices at Srikari Impetus solutions pvt Ltd. He is a Research Scholar under Dr.V.Vijaya Kumar Director -Centre for Advanced Computational Research (CACR) and Dr B Eswara Reddy professor of CSE Dept and principal of JNTU-A college of Engineering, Kalikiri,India from JNTU Kakinada. He received MTech(CS) from JNTU Hyderabad and MSc(Maths) from Osmania university, Hyderabad.

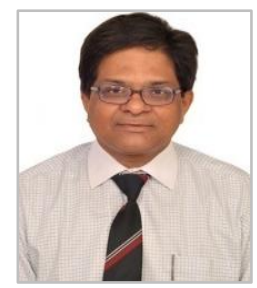

Dr. V. Vijaya Kumar is working as Director, Directorate of Admissions, Professor \& Head, Dept. of Computer Science, Rayalaseema University, Kurnool, India. Previously worked as Dean in Dept. of CSE \& IT, chairman Board of studies for CSE and IT and Director Centre for Advanced Computational Research (CACR) at Anurag Group of Institutions, (AGOI) (Autonomous), Hyderabad. He received integrated M.S.Engg, in CSE from USSR in 1989. He received his Ph.D. degree in Computer Science from Jawaharlal Nehru Technological University (JNTU), Hyderabad, India in 1998 and guided 28 research scholars for Ph.D. He acted as principle investigator for various $R \& D$ projects. His research interests include big data, image analytics, image retrieval, image processing, pattern recognition, digital water marking and cloud computing. He is the life member of CSI, ISCA, ISTE, IE (I), IETE, ACCS, CRSI, IRS and REDCROSS. He published more than 100 research publications till now in various national, international journals and conferences.

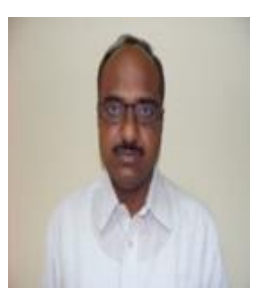

Dr. B. Eswara Reddy Graduated in B.Tech.(CSE) from Sri Krishna Devaraya University in 1995 . $\mathrm{He}$ received Masters Degree in M.Tech.(Software Engineering), from JNT University, Hyderabad, in 1999. He received Ph.D in Computer Science \& Engineering from JNT University, Hyderabad, in 2008. Currently, he is working as Professor of CSE Dept. \& Principal of JNTU-A College of Engineering, Kalikiri, India. He has published more than 100 publications in national and international conferences and journals. His research interests include Pattern Recognition \& Image Analysis, Data Mining, and Cloud Computing. He received UGC Major Research Project titled 'Cloud Computing Framework for Rural Health Care in Indian Scenario'. He is coauthor of the text books 'Programming with Java' (Pearson/Sanguine) and 'Data Mining' (Elsevier India). He is a life member of CSI, ISTE, ISCA, Fellow-IE (India) and member of IEEE.

How to cite this paper: MSLB. Subrahmanyam, V. Vijaya Kumar, B. Eswara Reddy, " A Robust Zonal Fractal Dimension Method for the Recognition of Handwritten Telugu Digits", International Journal of Image, Graphics and Signal Processing(IJIGSP), Vol.10, No.9, pp. 42-55, 2018.DOI: 10.5815/ijigsp.2018.09.06 